\title{
Somatotopic Organization of Single Primary Afferent Axon Projections to Cat Spinal Cord Dorsal Horn
}

\author{
Paul B. Brown, ${ }^{1}$ Wilbert E. Gladfelter, ${ }^{1}$ James C. Culberson, ${ }^{2}$ Dorothy Covalt-Dunning, ${ }^{3}$ Ramana V. Sonty, ${ }^{1}$ \\ Lillian M. Pubols, ${ }^{4}$ and Ronald J. Millecchia ${ }^{1}$ \\ Departments of ${ }^{\mathrm{P}} \mathrm{Phy}$ siology, ${ }^{2}$ Anatomy, and ${ }^{3}$ Biology, West Virginia University, Morgantown, West Virginia 26506 , and ${ }^{4} \mathrm{R}$. \\ S. Dow Neurological Sciences Institute, Good Samaritan Hospital, Portland, Oregon 97209
}

\begin{abstract}
Horseradish peroxidase injection of identified low threshold cutaneous mechanoreceptor (LTCM) primary afferent axons was used to assess the somatotopic organization of hindlimb projections to laminae III and IV of cat dorsal horn. Multiple injections in the same animals were used to assess bilateral symmetry and precision. Thirty-one axons were injected, with more than 1 axon injected in each of 8 animals (25 axons). Somatotopic relations between their receptive field (RF) centers and the centers of their dorsal horn projections were similar to the somatotopic relations between dorsal horn cell RF centers and cell locations. Very few reversals of mediolateral somatotopic gradients (proximodistal RF location as a function of mediolateral projection center) were observed. Two afferents with nearly identical RFs in 1 animal had nearly identical projections. These observations held for many different combinations of receptor types.

A simple mathematical model was used to demonstrate that assembly of dorsal horn cell RFs via passive sampling of the presynaptic neuropil by dorsal horn cell dendrites cannot account for the sizes of dorsal horn cell LTCM RFs. Hypothesized mechanisms for assembly of dorsal horn cell RFs must take into account the functional selectivity of connections required to produce RFs smaller than those predicted by the passive assembly model.
\end{abstract}

Fundamental questions concerning CNS topographic representations of sensory sheets (maps) include the nature of the information processing performed by such maps, the behavioral relevance of such processing, the physiological and anatomical substrates for such processing, and the developmental origins and subsequent maintenance of these substrates. We have been concerned in recent studies with the morphological correlates of somatotopic organization in the dorsal horn. The work reported here has determined the somatotopic organization, precision, and bilateral symmetry of projections of low threshold

Received Jun. 7, 1990; revised Aug. 30, 1990; accepted Sep. 13, 1990.

This research was supported by USPHS Grants NS12061, NS25238, and RR5433. Avonell Painter performed most of the histologic processing. Chantal Martineau, Richard Ammar, and Joseph Pollack provided valuable technical assistance. Stoelting Company provided generous support for development of electronics. Drs. H. R. Koerber and L. A. Ritz provided valuable advice in preparation of the manuscript.

Correspondence should be addressed to Dr. P. B. Brown, Physiology Department, West Virginia University Health Sciences Center, Morgantown, WV 26506. Copyright (c) 1991 Society for Neuroscience 0270-6474/91/010298-12\$03.00/0 cutaneous mechanoreceptor (LTCM) primary afferent terminal fields in the dorsal horn.

In 1975 Brown and Fuchs proposed that any region of the dorsal horn that contains cells excited by input from an area of skin receives some direct (monosynaptic) projections from that area of skin. Since dorsal horn cells are somatotopically organized in the horizontal plane, this would imply that the projections of cutancous axons are somatotopically organized in the same plane, in register with the dorsal horn somatotopy. We refer to this as the presynaptic somatotopy hypothesis. Brown and Culberson (1981) confirmed that dorsal roots project to the areas of dorsal horn in which their dermatomes are represented in the postsynaptic cell somatotopic map, supporting this hypothesis. Koerber and Brown (1980, 1982) showed that the projections of cutaneous nerves were more restricted in the horizontal plane than the projections of dorsal roots, as would be expected from the hypothesis. Whereas adjacent dorsal roots have overlapping dermatomes and dorsal horn projections, cutaneous nerves typically have nonoverlapping LTCM innervation and projection fields (at least for A fibers: Koerber and Brown, 1980, 1982). Dorsal horn projections of cutaneous nerves are also smaller than those of dorsal roots, as would be expected from the fact that cutaneous nerve innervation fields are smaller than dermatomes. The work of Ritz et al. (1985, 1989) demonstrated the presynaptic somatotopy of trunk and tail afferents and showed that LTCM axons have both crossed and uncrossed collateral branches that convey their input to dorsal horn areas where such axons contribute to the crossed and uncrossed components of dorsal horn cell receptive fields (RFs).

It is possible that sorting at the level of precision of peripheral nerve projections is adequate for the assembly of the dorsal horn cell map of the skin. In this case, it could be expected that there would be little additional precision in the termination patterns of primary afferents, and a single afferent could project to a large portion of its cutaneous nerve's projection field. In addition, the serial order of projections along a trajectory across the map need not exactly match the serial order of the axons' RFs across the skin. A degree of selectivity in the connections of afferents to cells could still provide the acuity inherent in dorsal horn cell RFs, and the orderliness of the dorsal horn cell somatotopic map.

On the other hand, if dorsal horn cells assemble their RFs by passive sampling of a somatotopically ordered presynaptic neuropil, then LTCM terminal fields must form a much more precise pattern than the projections of peripheral nerves. Given the relatively large dorsal horn cell RFs and the pattern of shifting overlap in their somatotopic organization, the terminal fields 
of primary afferents with contiguous RFs still need not be segregated into contiguous projection zones. Instead, the projection centers of axons whose RFs describe a continuous locus across the skin should display relatively orderly trajectories across the map, and contiguous RFs could be associated with overlapping projections. A limited amount of noise in the organization of the presynaptic neuropil could be tolerated: the scatter of projection centers and sizes of projections would simply have to fall within limits demanded by the convergence ratios implied by the ratios of dorsal horn cell RF areas to axon RF areas.

Judging from the precision of projections of cutaneous nerves, where homologous pairs on opposite sides of the body have projections that are congruous within about $100 \mu \mathrm{m}$ (see the figures in Koerber and Brown, 1980, 1982), it would seem likely that primary afferents would have at least a similar precision (e.g., the projections of axons with congruous RFs would be congruous within $\pm 100 \mu \mathrm{m}$ or less). Data from the first injection studies of LTCMs (A. G. Brown et al., 1977a, 1978, 1980a, 1981) suggest that primary afferent projections are more restricted mediolaterally and rostrocaudally than the projections of cutaneous nerves, but it is not possible to determine the details of somatotopy from those reports. Preliminary horseradish peroxidase (HRP) injection studies by R.E.W. Fyffe, P.B. Brown, L.M. Pubols, and A.G. Brown (unpublished, 1980-1981) suggested that the projections of single LTCMs in the horizontal plane carried somatotopic precision of afferent terminal arbors to a finer grain than peripheral nerves.

The present study was undertaken to evaluate systematically the somatotopic organization of individual LTCM axon projections to dorsal horn, to obtain preliminary information on the precision and bilateral symmetry of such projections, and to test the simplest model of assembly of dorsal horn cell RFs. The results of this investigation confirm that primary afferent termination fields are qualitatively in register with the dorsal horn cell somatotopic map, and that there is a high degree of bilateral symmetry of projections to right and left dorsal horns. However, projections are not sufficiently precise for a model of dorsal horn cell RF assembly based on passive sampling of the presynaptic neuropil by dorsal horn dendrites.

Preliminary reports of this work have been published elsewhere (P. B. Brown et al., 1988; Sonty et al., 1988).

\section{Materials and Methods}

Subjects were mongrel cats, $2-4 \mathrm{~kg}$, of either sex, obtained from pounds and USDA-licensed dealers. Animals were first anesthetized with either halothane or a mixture of xylazine $(0.5 \mathrm{mg} / \mathrm{kg})$ and ketamine $(20 \mathrm{mg} /$ $\mathrm{kg}$ ). Tracheal, carotid and jugular cannulae were installed, for artificial respiration, blood pressure monitoring, and infusion of liquids and drugs, respectively. When halothane was used to induce anesthesia, it was discontinued at this point, and replaced with alpha-chloralose $(70 \mathrm{mg}$ $\mathrm{kg}$ ). When ketamine and xylazine were used, chloralose was administered in a series of supplements as the original anesthetic wore off. Surgical anesthesia was maintained with supplements of $10-20 \%$ of the initial chloralose dose, whenever nociceptive reflexes could be detected, the pupils were responsive to light, or the blood pressure responded to noxious stimuli. A rectal probe provided feedback control for a temperature-controlled heating pad and infrared lamps, used to maintain core temperature at $38^{\circ} \mathrm{C}$.

The animal was mounted in a spinal frame and headholder, in traction, on a vibration isolation table. The lumbar enlargement was exposed and covered with mineral oil. The segment chosen for recording was raised on a platform. A bilateral pneumothorax was performed, and the animal was paralyzed with gallamine triethiodide (initial dose, $20 \mathrm{mg}$, supplemented with 10-mg supplements every half hour). Endexpiratory $\mathrm{CO}_{2}$ was maintained at $3.5-4.0 \%$ with a respirator. During paralysis, pupillary reflexes and blood pressure responses to noxious stimuli were used to assess anesthetic level, and the paralyzing agent was allowed to wear off occasionally to test withdrawal reflexes.

Microelectrodes were filled with $30 \% \mathrm{HRP}$ in $0.1 \mathrm{M}$ phosphate buffer, $\mathrm{pH} 7.6$, and $0.2 \mathrm{M}$ or $0.5 \mathrm{M} \mathrm{KCl}$. These electrodes were broken back to 15-35 M $\Omega(<1.5 \mu \mathrm{m})$. A high input impedance differential amplifier was used for recording; microelectrodes were advanced through the white matter overlying the dorsal horn in $300 / \mathrm{sec}$ bursts of 81 -micron steps with a direct-drive stepping motor microdrive.

If manual exploration of the leg revealed that a penetrated element was a LTCM axon, its receptor type and RF were determined with a variety of hand-held stimuli, according to established criteria (Horch et al., 1977). An axon was injected if (a) it was thought not likely to overlap the projection of a previously injected axon, (b) the resting potential was at least $-50 \mathrm{mV}$, (c) the action potentials were at least $50 \mathrm{mV}$, and (d) the penetration remained stable after a few cycles of HRP injection.

Two cascaded pulse amplifiers (Stoelting Company) were used to provide positive dc $(20 \mathrm{nA})$ and pulsatile $(20 \mathrm{nA})$ currents, under control of a stimulator/laboratory controller (Millecchia and Brown, 1986; Brown and Millecchia, 1987). Each injection lasted $1 \mathrm{~min}$, using $375-\mathrm{msec}$ pulses recurring every $500 \mathrm{msec}$ (injected charge $20 \mathrm{nA} \cdot \mathrm{min}$ sustained plus $15 \mathrm{nA} \cdot \mathrm{min}$ intermittent). Usually it was possible to maintain a stable penetration if the resting and action potentials returned to normal after a $1-$ min injection. Total HRP injection for successful staining was usually in excess of $100 \mathrm{nA} \cdot \mathrm{min}$. Axons were injected, on alternating sides, at a series of rostrocaudal locations spanning the segment on the platform, and far enough apart to prevent overlapping terminal arbors. With experience, it was possible to place injections closer together, reconstructing multiple injections on the same side by tracing collaterals through their parent axons to injection sites. In cases where this approach was unsuccessful and projections could not be separated unambiguously, the data were not used. Empty marker electrodes were placed on the cord midline at the rostrocaudal levels of injection sites. When time permitted, other segments were explored.

The cat was kept deeply anesthetized, and $6 \mathrm{hr}$ after the last dye injection, it was perfused with $500 \mathrm{ml} 0.9 \% \mathrm{NaCl}$ in water with $0.1 \mathrm{~g}$ procaine hydrochloride and $0.1 \mathrm{~g} \mathrm{NaNO}$ at $38^{\circ} \mathrm{C}$, followed by 1 liter of $0.75 \%$ glutaraldehyde and $0.5 \%$ paraformaldehyde in $0.1 \mathrm{M}$ phosphate buffer, $\mathrm{pH} 7.4$, at approximately $24^{\circ} \mathrm{C}$.

Tissue blocks were soaked overnight in sucrose-saturated $0.1 \mathrm{M}$ phosphate buffer (pH 7.4) at $4^{\circ} \mathrm{C}$, and transverse sections were cut at $50 \mu \mathrm{m}$ on a freezing microtome, and processed with the cobalt-enhanced diaminobenzidine method (Adams, 1977). All sections were mounted and examined for filled profiles. A modified Dorsalview program (P. B. Brown, 1986) was used to analyze the data.

\section{Results}

A total of 31 axons was injected adequately for analysis. An axon was judged to be adequately stained if the most rostral and caudal well-stained collaterals had relatively few boutons $(<30)$, indicating that any more rostral or caudal collaterals which might have been poorly stained would have still fewer boutons (viz., Koerber et al., 1990, for details of bouton distributions of injected axons). The sample included 12 slowly adapting type I (SAI), 4 slowly adapting type II (SAII), 12 hair, 2 Pacinian corpuscle (PC), and 1 rapidly adapting glabrous (RAG) axon which were adequately filled and had projection fields in laminae III and IV, separable from other afferent projections (in cases of multiple injections in single animals).

For a few axons, the distribution of boutons (presumed sites of synaptic transmission: e.g., Maxwell et al., 1982) was plotted on camera lucida outlines of laminae III-IV. Sets of 10 adjacent sections wcre supcrimposcd in order to assess the mediolateral and dorsoventral extent of the bouton distribution in $500-\mu \mathrm{m}$ lengths of dorsal horn. One such axon is illustrated in Figure 1. The tracings of sections were manually positioned so that laminae I-IV (outline) were as well aligned as possible. Boutons were then plotted as dots. The clouds of boutons formed a mediolaterally narrow wedge spanning laminae III and IV. This approximates a radial sheet: the axis of the sheet coincides with 

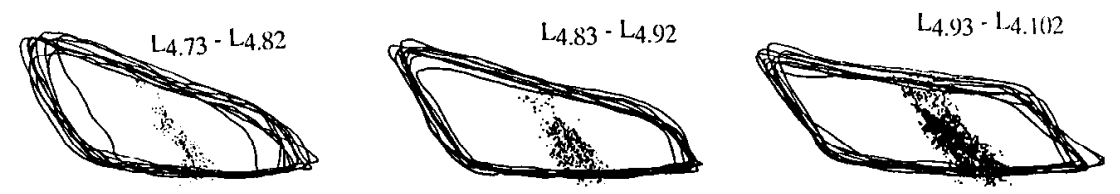

Figure 1. Bouton distribution of a single SAI afferent projection. Each drawing represents 10 superimposed serial transverse sections (each section $50 \mu \mathrm{m}$ thick), manually oriented so that laminae III-IV (outline) are aligned. Dots represent boutons visible under $100 x$ magnification. Thus each drawing can be viewed as a view of a cloud of boutons suspended in a $0.5-\mathrm{mm}$ long tunnel formed by the space occupied by laminae III and IV, looking from the caudal end of the tunnel toward the rostral end. $\mathrm{L}_{4 . x}-\mathrm{L}_{4, y}$ designations indicate segment $\mathrm{L}_{4}$, sections $x-y$. Thus, $\mathrm{L}_{4.73}-\mathrm{L}_{4.82}$ would span the rostrocaudal dimension from section 73 through section 82 of segment $L_{4}$, where sections are numbered from the rostral to the caudal end of the segment.
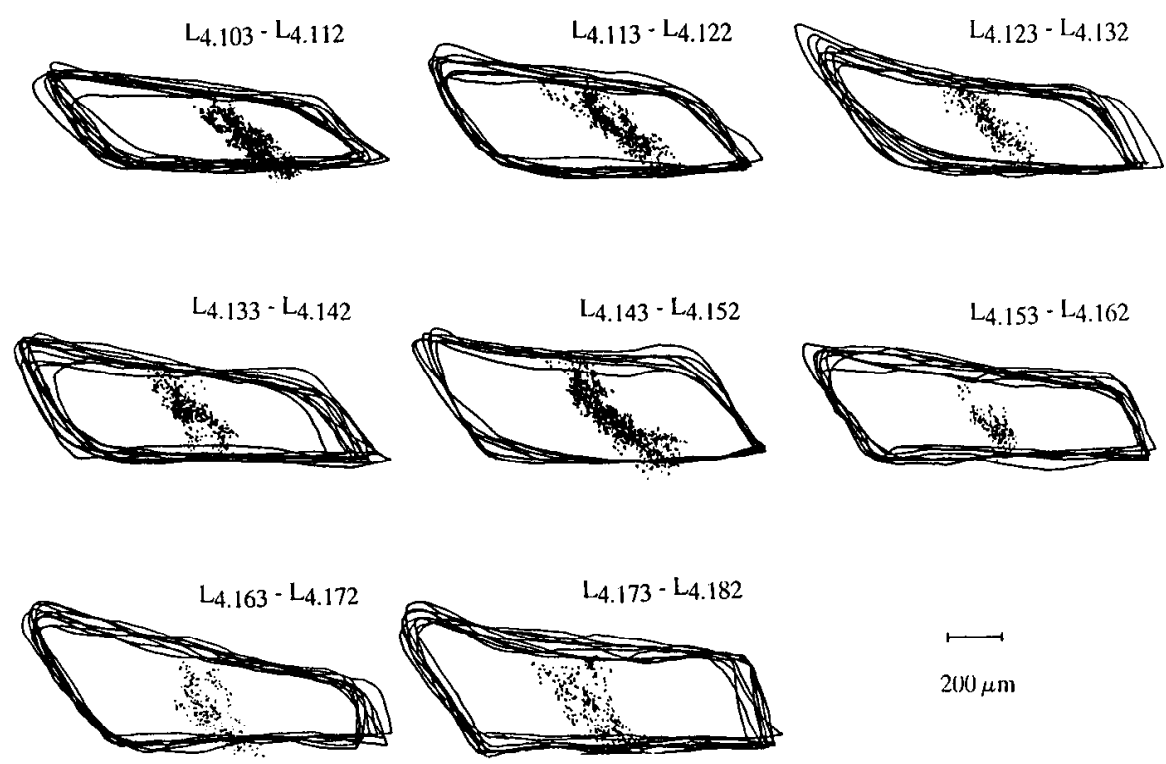

the neutral axis of somatotopy for dorsal horn cells (e.g., Brown and Fuchs, 1975), cutaneous nerves (Koerber and Brown, 1980, 1982), and current source density distributions of monosynaptic EPSPs evoked by single action potentials in single SAI axons (Brown and Ritz, 1986).

Since the somatotopic map is in the plane of the laminae, we have visualized bouton distributions as projections in the horizontal plane (dorsal views: e.g., Brown and Fuchs, 1975; A. G. Brown et al., 1980b; P. B. Brown, 1986). Mediolateral bouton distributions in laminae III-IV were measured manually using a nomogram and camera lucida. Small random variations of the manual orientation of the nomogram caused scatter in the mediolateral localization of boutons from section to section. This scatter was greater than that observed in the transverse sections of Figure 1 and in previous studies using computerautomated methods (R. E. W. Fyffe, A. G. Brown, L. M. Pubols, and P. B. Brown, unpublished). Therefore the scatter was reduced by a smoothing procedure. Figure 2 illustrates this smoothing process. In Figure 2, $A$, the mediolateral distributions of boutons are plotted in an uncorrected dorsal view (width of dorsal view is normalized), where each horizontal line represents the mediolateral extent of boutons in 1 section, as measured with the nomogram. In this, and all other dorsal views, the view is of a left dorsal horn, with the medial edge of laminae III-IV to the right, and the lateral edge to the left (data obtained from right dorsal horns were rotated $180^{\circ}$ about the longitudinal axis). This is the axon of Figure 1; note the greater scatter in Figure 2, $A$ than in Figure 1. In Figure 2, $B$, the axis of the uncorrected projection is plotted, by a line connecting the centers of bouton distributions, section by section. This axis was then spatially low-pass filtered by computing the weighted average of each center and $n$ adjacent centers in each direction:

$$
\bar{x}_{j}=\frac{x_{j}+\sum_{i=1}^{n} \frac{x_{j+i}+x_{j-i}}{i+1}}{1+\sum_{i=1}^{n} \frac{2}{i+1}}
$$

where $\bar{x}_{j}$ is the weighted average for section $j$, and $x_{j+i}$ is the uncorrected center location for section $j+i$, excluding sections with no boutons.

The number of sections on each side of the center section, $n$, was adjusted to produce a degree of scatter similar to that in Figure 1: $n=5$. The smoothed axis is illustrated in Figure 2, $D$. The mediolateral distributions of boutons were then replotted, centered on the smoothed centers (Fig. $2 \mathrm{C}$ ). The figure is organized so that original and smoothed distributions of boulons and centers can be compared, one above the other ( $A$ and $C, B$ and $D$ ). Note that the dorsal view in Figure 2, $C$, more closely approximates the mediolateral extent of boutons in the camera lucida drawings of Figure 1.

Bilateral symmetries of dorsal horn cell somatotopy (P. B. Brown et al., 1990), and peripheral nerve projections (Koerber and Brown, 1980, 1982) suggest that a similar bilateral symmetry might exist in the projections of individual axons. Therefore, we compared projections of axon pairs on opposite sides of the cord, using normalization of laminae III-IV width. Multiple axons were injected in each of 8 animals ( 25 axons). Figures 3 and 4 illustrate the results. In these figures, dorsal views are normalized on plots using average widths and lengths of segments across all animals. A figurine is drawn next to each projection, with the RF location indicated by a dot. Afferent type 
Table 1. Comparisons of RFs and projections of pairs of axons, where each pair has projections to the same rostrocaudal level in the same animal

\section{Figure Comparison}

$3 A$ mid-thigh $\mathrm{RF}$ projects lateral to knee $\mathrm{RF}$

$3 B \quad$ knee RF projects lateral to toe $2 \mathrm{RF}$

$3 C$ hip RF projects lateral to toe $4 \mathrm{RF}$

$3 D$ proximal thigh $\mathrm{RF}$ projects lateral to foot $\mathrm{RF}$

$4 A$ proximal foot RF projects lateral to mid-foot RF

$4 A \quad$ overlapping thigh RFs, overlapping projections

$4 B$ toe $5 \mathrm{RF}$ projects lateral to toe $4 \mathrm{RF}$

$4 B$ mid-thigh RF projects lateral to ankle RF

$4 C$ toe $5 \mathrm{RF}$ projects lateral to and overlapping projection

from RF lateral to footpad

$4 D \quad$ ankle RF projects lateral to proximal foot $\mathrm{RF}$

$4 D$ proximal foot RF projects lateral to mid-foot RF

4D mid-foot RF projects lateral to and overlapping projection from toe $5 \mathrm{RF}$

$4 D$ ankle RF projects lateral to mid-foot RF

$4 D$ ankle RF projects lateral to toe $5 \mathrm{RF}$

$4 D$ proximal foot RF projects lateral to toe $5 \mathrm{RF}$

and the side of the cord ( $\mathrm{L}$ or $\mathrm{R}$ ) are also identified. Figures 3 and 4 represent projections of multiple LTCMs in single animals; in Figures 3 and 4 each diagram represents 1 animal. Figure 5 depicts the 6 cases where single axons were labeled, in 2 composites. We concentrate here on comparisons of axon pairs with projections to the same rostrocaudal level in the same animal, comparing the relations between RFs and the projections on the mediolateral axis of laminae III and IV.

Table 1 summarizes comparisons made in Figures 3 and 4. There is 1 case where RFs of 2 axons are very similar, and their projections overlap. There are 12 cases (including 4 axons considered in 6 paired combinations) in which the more proximal RF is associated with a more lateral projection. In 1 case, toe 5 projects lateral to toe 4 , and in 1 case skin next to the central footpad projects medial to toe 5 . Thus 13 of 15 pairs ( 9 of 11 independent pairs) obey the rule that relatively distal RFs project medial to relatively proximal RFs. Discounting the 1 tie, agreement among 9 of 10 independent pairs is statistically significant ( $\mathrm{df}=1, \chi^{2}=6.4, p<0.02$ ). The 1 apparent anomaly, in which an SAI lateral to the central footpad projected medial to a RAG on toe 5 , is probably a genuine reversal of the distoproximal somatotopic gradient, which is normal (Wilson et al., 1986; B. H. Pubols et al., 1989; L. M. Pubols, unpublished observations; P. B. Brown, unpublished observations).

In Figure 5, there is 1 reversal of the somatotopic gradient, among a smaller number of independent pairs. The only difference between Figures 3 and 4 and Figure 5 is that each plot in Figures 3 and 4 is of multiple axons in a single animal, and in Figure 5 each plot is a composite from more than 1 animal. Therefore the 1 apparent reversal of trajectory in Figure 5 is probably due to interanimal variation, which has already been documented in terms of dorsal horn physiology (e.g., Brown and Fuchs, 1975), projections of dorsal roots (e.g., Brown and Culberson, 1981), and projections of cutaneous nerves (e.g., Koerber and Brown, 1980, 1982).

Unfortunately, we cannot use our sample of 25 pairs to estimate the precision and bilateral symmetry of axon projections. If we could assume that there is an invariant underlying function

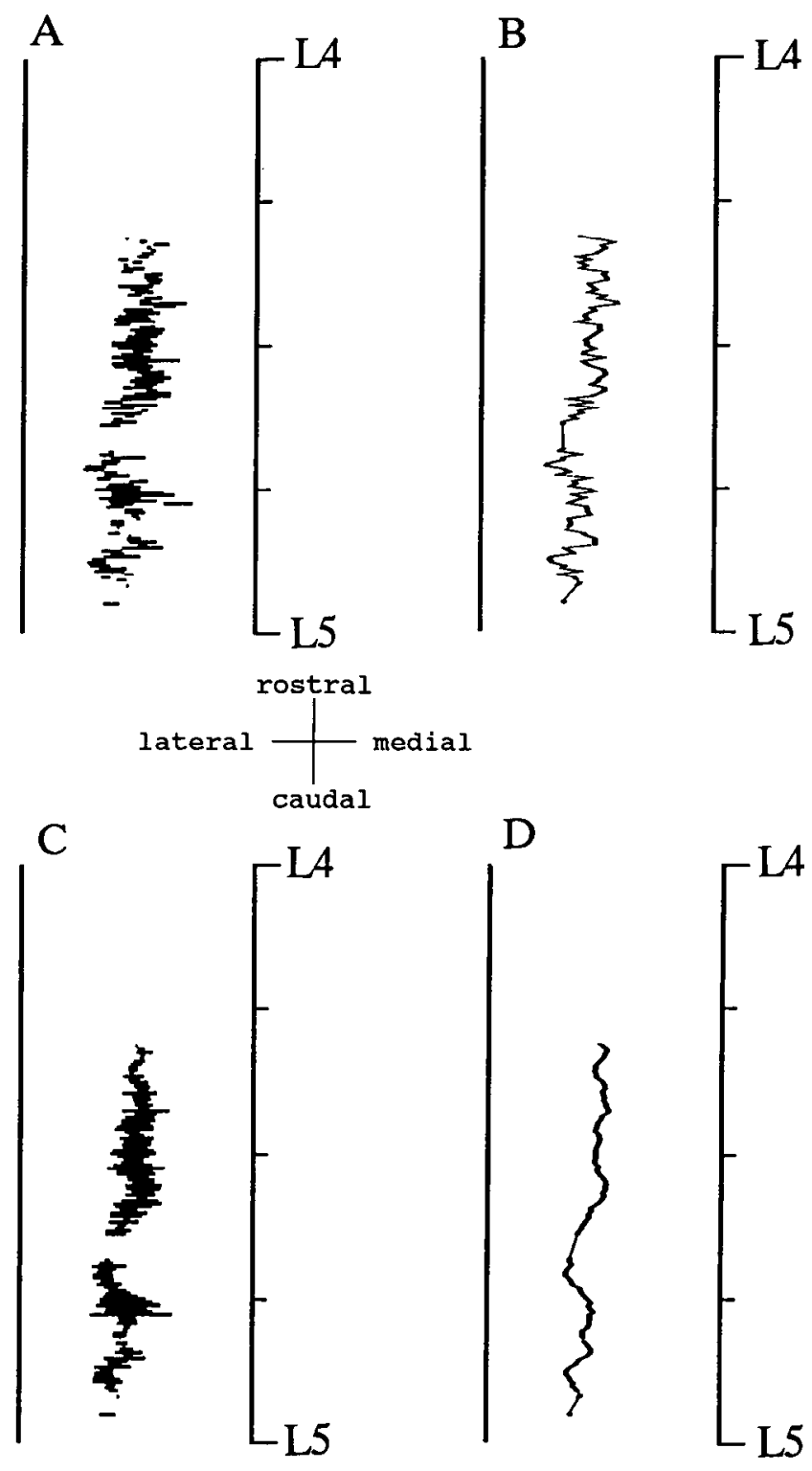

Figure 2. Correction for random scatter in dorsal views caused by errors in manual alignment of nomogram used to measure medial and lateral edges of bouton distributions. $A$, Dorsal view (horizontal projection), with normalized segmental lengths and widths, unsmoothed data (raw values of mediolateral positions of medial and lateral boundaries). Each horizontal line is the mediolateral distribution of boutons in 1 section. In this, and in all other dorsal views, the view is of a left dorsal horn, with lateral to the left, medial to the right, rostral to the top, and caudal to the bottom. Data from the right side in all figures are left-right inverted. $B$, Dorsal view of the centers of projections on all sections, connected by straight lines. $C$, Corrected dorsal view with each section's bouton distribution centered on the corrected ("smoothed") center for that section. $D$, Corrected centers, derived from $B$ using the spatial smoothing algorithm described in text.

that describes mediolateral projection location as a function of proximodistal RF location, we could measure the variance in projection location about this function. However, we know that such a function varies from 1 rostrocaudal location to another within an animal, and from 1 animal to another, from inspection of Figures 3-5. Nevertheless, there was 1 case in which the RFs of 2 axons on opposite sides of the same animal were essentially identical. It is instructive to examine the agreement between the 2 projections in this case (Fig. $4 A$ : proximal thigh). The com- 

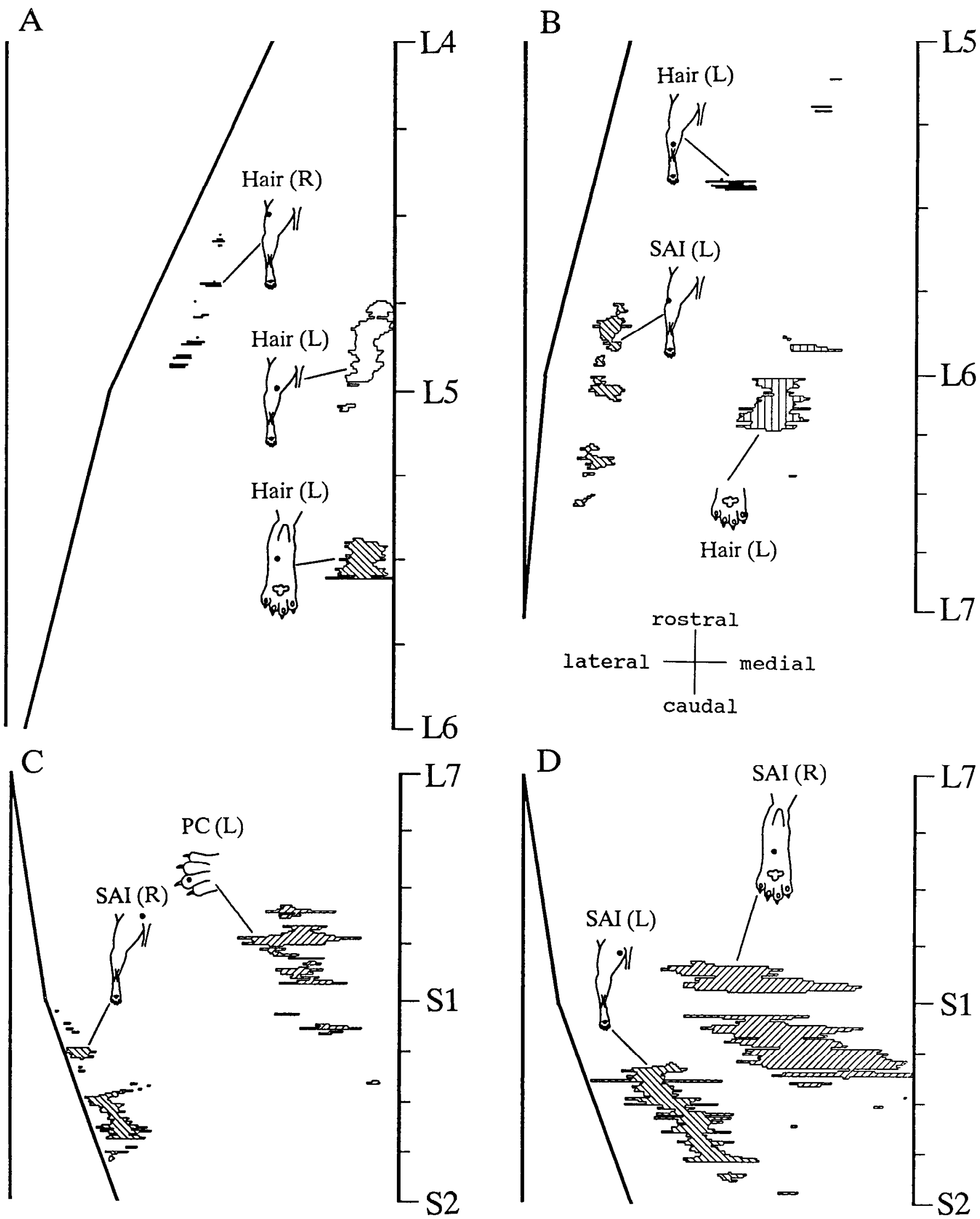

Figure 3. Dorsal views of multiple axon projections, 1 animal per diagram. In each animal, more than 1 axon was injected with HRP, after identifying the receptor type and receptive field location. Data from both left and right dorsal horns are superimposed after left-right inversion of right dorsal horn data. Segments are drawn using lengths and widths that are averaged across animals. Each axon within an animal has a different cross-hatching pattern. Figurines indicate RF locations (drawn as dots indicating RF centers), labels indicate afferent types: $S A I$, slowly adapting type I; $S A I I$, slowly adapting type II; $P C$, Pacinian corpuscle; and $L$ or $R$ indicates axon was on left or right side. 

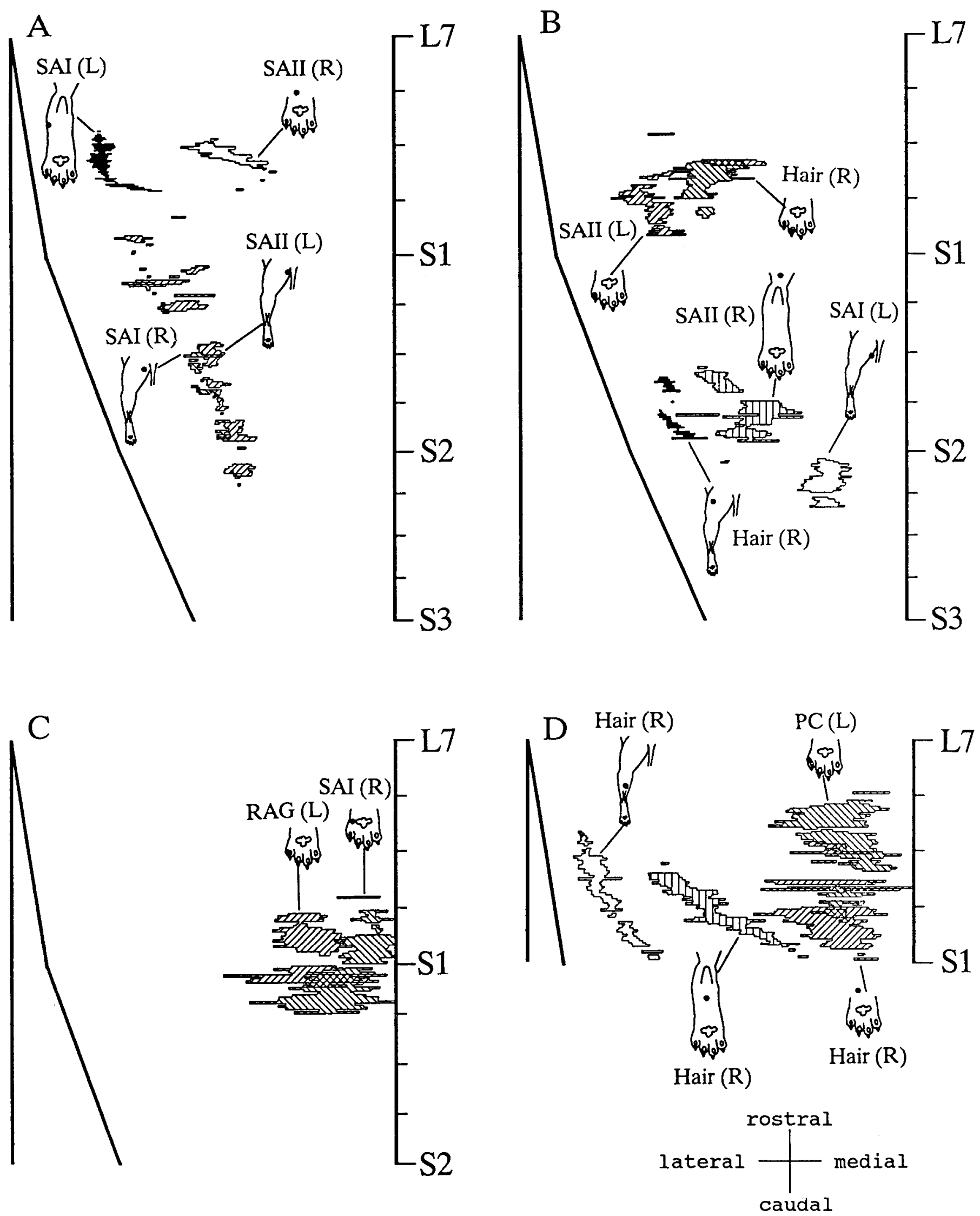

Figure 4. Dorsal views of multiple axon projections, for four more animals. See Figure 3 legend for details of figure conventions. $R A G$, rapidly adapting glabrous. 


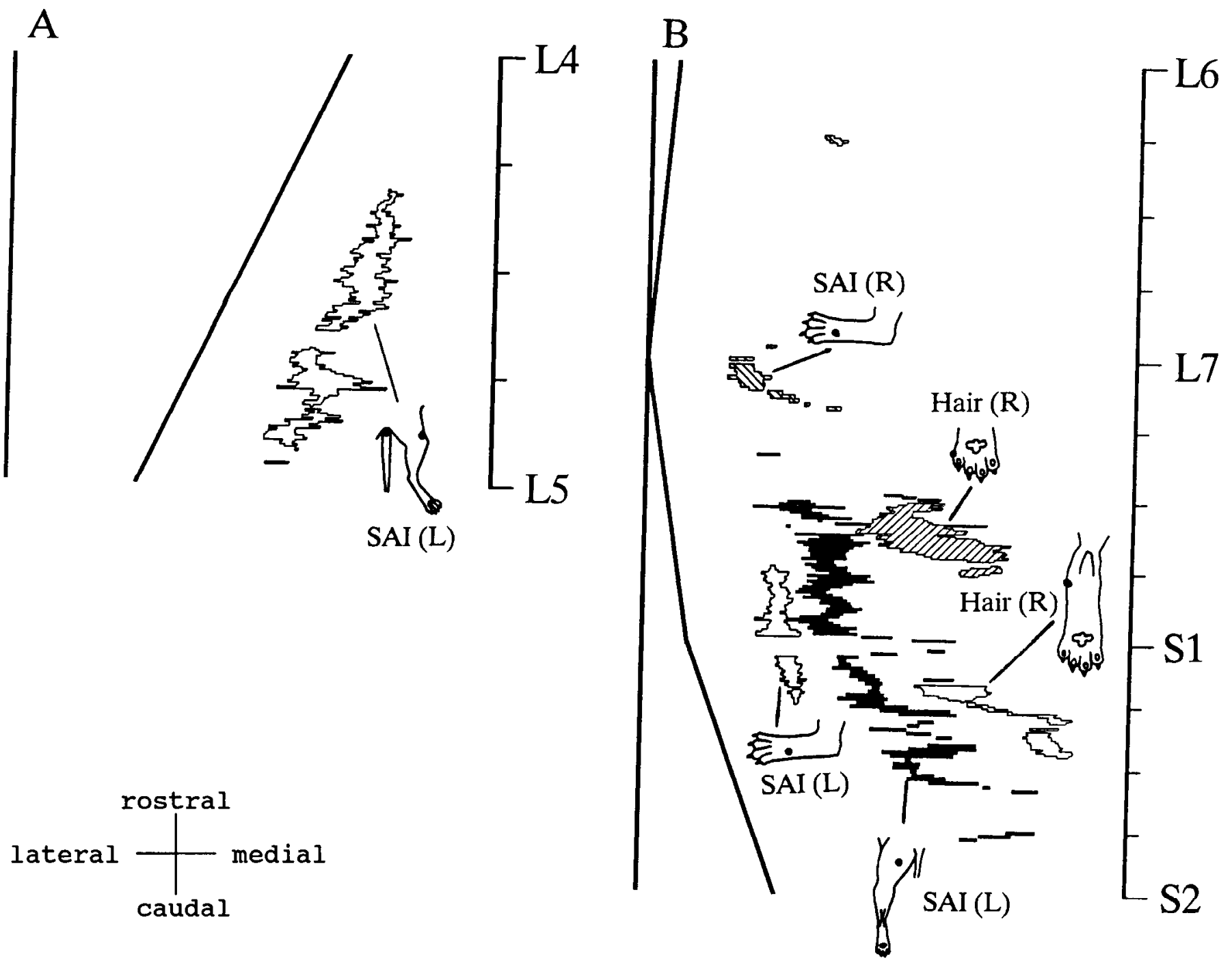

Figure 5. Six axon projections in 6 animals, combined in 2 dorsal views. See Figure 3 legend for details of figure conventions. Note that in Figures 3 and 4 , each drawing portrays data from a single animal. In this figure, to save space, data from multiple animals are combined in each figure.

puted centers of the bouton distributions differed by only $4 \%$ of the dorsal horn width ( $32 \mu \mathrm{m}$ at this rostrocaudal level, where the dorsal horn is $800 \mu \mathrm{m}$ wide) mediolaterally. The centers differed by only 3 sections ( $150 \mu \mathrm{m}$, or about $2.5 \%$ of the lengths of the projections) rostrocaudally. The 32- $\mu \mathrm{m}$ error mediolaterally is the root mean square of the average measurement error, bilateral asymmetry, and variation in the placement of the projection center in the map. We cannot separate asymmetry and placement errors, but we can estimate their combined error by deducting a reasonable amount for measurement error. If we assume a random measurement error of $10 \%$ from 1 section to the next, over 125 sections, the measurement error of the computed center is $10 / \sqrt{125}=0.89$ or $0.7 \mu \mathrm{m}$. Therefore the measurement error makes a negligible contribution to the calculated centers, and the combined placement and asymmetry error is on the order of $\pm 32 \mu \mathrm{m}$. This single pair therefore exhibits a remarkable degree of precision in the locations of their projection centers, only slightly larger than a dorsal horn cell and smaller than a cell's dendritic tree. Interestingly, their average widths and lengths are not so precise. Their lengths differ by 13 sections $(650 \mu \mathrm{m})$, or $10 \%$ of their average length $(13 \%$ of the length of $\left.\mathrm{S}_{1}\right)$. Their widths differ by $39 \mu \mathrm{m}(5 \%$ of the width of the dorsal horn, and $20 \%$ of their average width).

The data of Figures 3-5 clearly show that the distributions of afferent terminals are not as stereotyped as suggested by some previous reports. Rather than relatively uniform sagittal sheets, the terminal fields form ribbons of varying length and width, and many display a curvature that is quite pronounced when displayed on an appropriate scale. We shall defer analysis of variations in terminal field geometry to a later paper, where we shall show that such variations are a consequence of the varying scale (magnification) of the somatotopic map.

Our data are not sufficiently extensive to determine whether there exist quantitative differences in the somatotopic organization of different afferent types. However, since our dorsal views are based on combined plots of several afferent types and no obvious reversals occur in mediolateral somatotopic gradients within single animals, the somatotopic organization appears to be qualitatively similar for different afferent types.

Figure 6 summarizes the somatotopic organization of all 31 afferents in a single composite figurine map, plotting each RF (filled circle) at the location of the center of the corresponding 


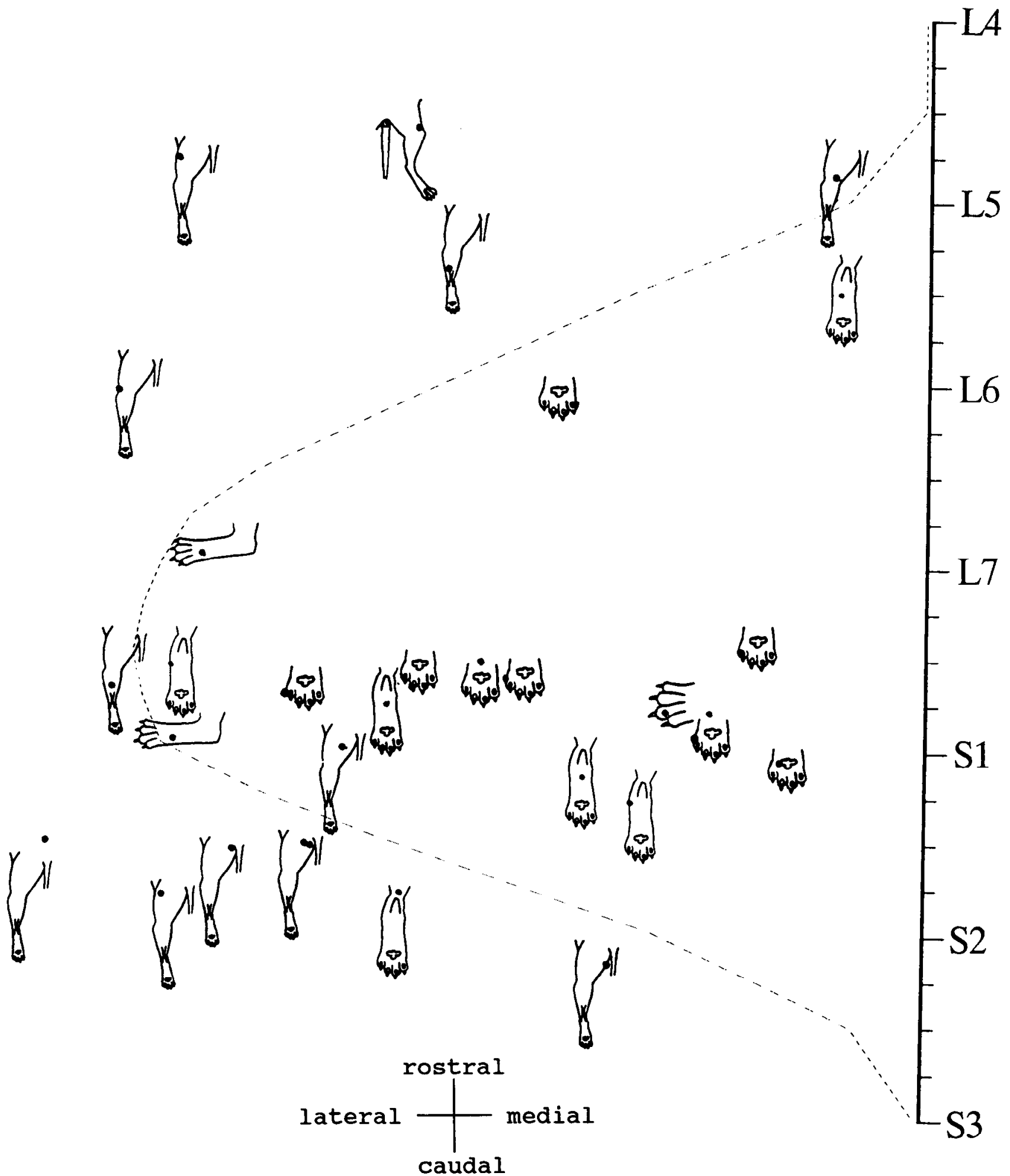

Figure 6. Summary diagram of somatotopic organization of projections of all 31 axons, on a single dorsal view, regardless of receptor type. The figure was compiled by plotting RFs (dots on figurines) at geometric centers of corresponding axon projections, in a left-sided dorsal view. Normalized lengths and widths of segments. Note that additional gradicnt reversals (apparent discrepancies in the mediolateral gradient of somatotopy) are introduced by combining data across animals.

afferent's projection. Note that, although some apparent reversals of mediolateral trajectories occur as a result of interanimal variation, the general organization of the presynaptic neuropil is very similar to that presented by a number of laboratories for dorsal horn cclls. The prepostaxial, proximodistal, and embry- ologically ventrodorsal gradients seen in dorsal horn cell somatotopy (Wall, 1960; Bryan et al., 1973; Brown and Fuchs, 1975; Light and Durkovic, 1984; Wilson et al., 1986) are present in the presynaptic termination patterns. The foot region of the map is approximately outlined in Figure 6 by the broken line. 
A

Figure 7. Test of passive assembly model of dorsal horn cell receptive field assembly (see text). $A$, Average sizes of LTCM afferent axon RFs are lined up edge-to-edge along the length of the leg. $B$, Average sizes of dorsal horn cell low threshold mechanoreceptive fields, lined up edge-to-edge along the length of the leg. $C$. Average primary afferent projections zones of single axons, lined up edge-to-edge across the dorsal horn. Since slightly more than 4 axon projection zones can be accommodated, approximately $4-5$ contiguous axon RFs havc ovcrlapping representation in the presynaptic bouton distributions. $D$, Average modeled dorsal horn cell RFs lined up edge-to-edge along the length of the leg, as predicted by the passive assembly model, assuming zero dendritic tree widths. Since slightly more than 4 axon terminal distributions can be accommodated across the width of the dorsal horn, the model predicts that only slightly more than 4 dorsal horn cell RFs can be accommodated along the length of the leg.
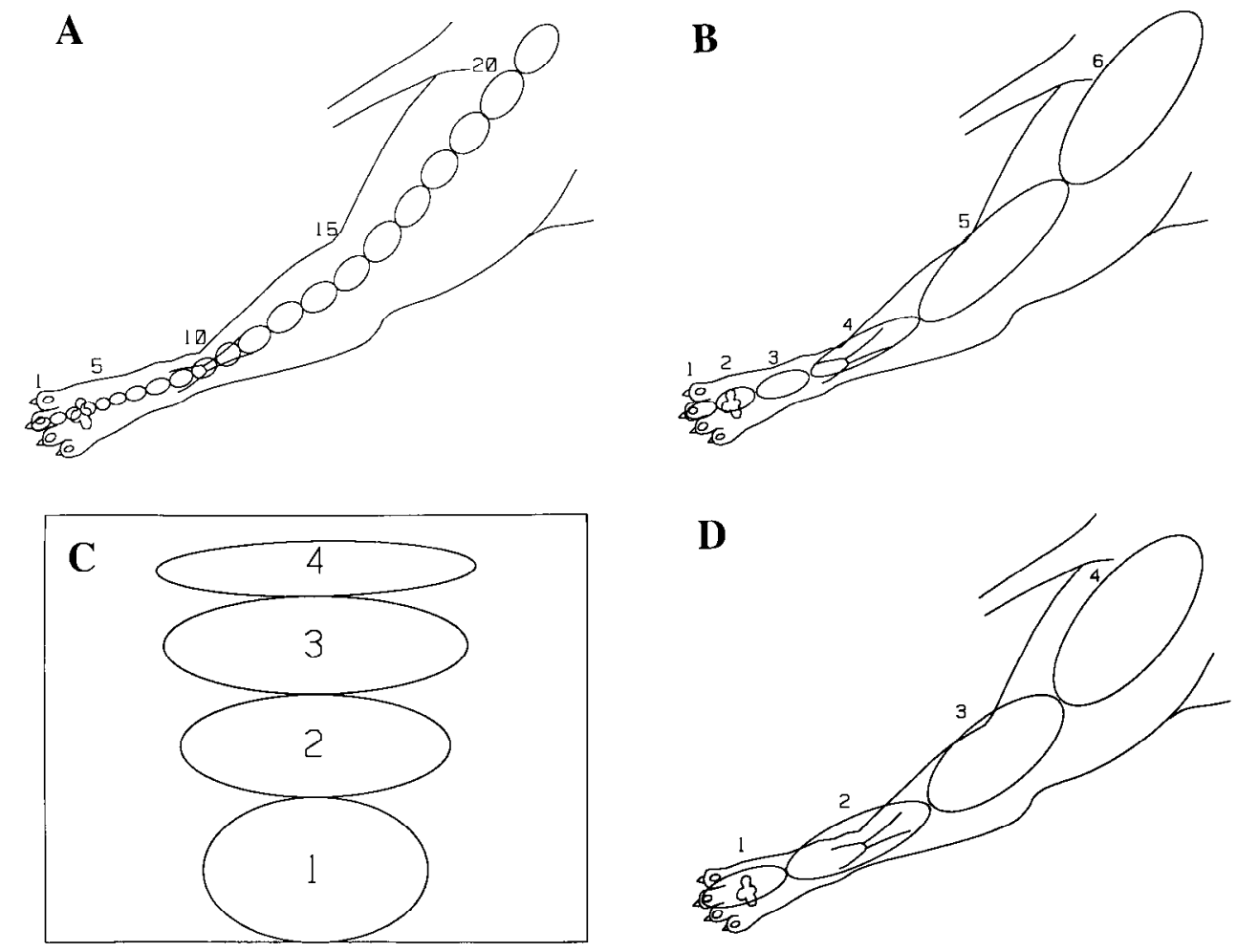

This region is very similar to that described for dorsal horn cells. In comparison with the representation of the rest of the leg, the image of the foot on the dorsal horn map is greatly magnified. Although the toe representation is not outlined, it is clear that the magnification or scale factor for the toes is even greater than for the rest of the foot. These features are also similar to the dorsal horn cell somatotopic map.

The data presented here, on the projections of single afferents to the dorsal horn, make it possible for the first time to examine the feasibility of the simplest model of dorsal horn cell RF assembly, in which each cell passively samples primary afferent terminals in the region of neuropil penetrated by its dendrites. This model assumes that primary afferent axons lay down a presynaptic neuropil in which their boutons are somatotopically organized, and cells' RFs are assembled by unselective contacts between their dendrites and afferent axon boutons available to them. Thus, each cell's RF would consist of the aggregate of all RFs of all the afferent axons whose boutons are intermingled with the cell's dendrites. This requires a sufficient degree of precision of bouton and dendrite distributions that no somatotopically inappropriate connections can be made. We refer to this as the passive assembly model, and we shall concentrate on the mediolateral axis of the dorsal horn, which maps the distoproximal axis of the leg. Figure $7, A$, indicates the average sizes of LTCM RFs lined up edge-to-edge along the length of the leg (based on data presented in Brown and Koerber, 1978). Figure $7, B$, illustrates the average lengths of dorsal horn cell LTCM excitatory RFs (based on data presented in P B. Brown et al., 1975). In this figure, it is clear that the spatial resolution permitted by RF size decreases after the first synapse, going from about 20 RFs along the length of the leg for primary afferents to about 6 RFs for dorsal horn cells, or a convergence ratio along the distoproximal axis of the leg of about 3.3 con- tiguous primary afferent RFs per dorsal horn cell RF. We shall refer to this as the contiguous convergence ratio. A correct model of dorsal horn cell RF assembly must predict a similar contiguous convergence ratio.

Such a model must take into account the mediolateral extents of both primary afferent terminal fields and of dorsal horn cell dendrites. Adequate data for dorsal horn cell dendrites are not available, but even if we assume the limiting case of a single dorsal dendrite with zero mediolateral width for each dorsal horn cell, we can demonstrate that the convergence from primary afferents is too high to predict actual dorsal horn cell RF lengths. In this limiting case, assuming no scatter in the locations of primary afferent projections, the condition for assembly of contiguous dorsal horn cell RFs is that their dendrites must be spaced 1 primary afferent terminal field width apart. Dendrites any closer together would overlap the same afferent axon bouton distribution, would have that axon's RF in common, and would therefore have overlapping RFs. Therefore, the number of dorsal horn cell RFs that can span the length of the leg should be the same as the number of primary afferent terminal fields that can span the width of the dorsal horn. Contiguous terminal fields of average width are plotted in Figure $7, C$. Slightly more than 4 contiguous terminal fields can, on the average, span the width of the dorsal horn. Therefore the passive assembly model predicts that only slightly more than 4 dorsal horn cell RFs can span the length of the leg (Fig. $7 D$ ), for a contiguous convergence ratio of about 4.5 . If the widths of dorsal horn cell dendrites were taken into account, the number of contiguous dorsal horn cell RFs would decrease and the contiguous convergence ratio would increase. In addition, noise in the presynaptic somatotopy would further increase this ratio. If the model were to take all these factors into account, the predicted contiguous convergence ratio would probably lie somewhere between 5 and 7 . Finally, 
these calculations were performed only for the average observed dorsal horn cell RF lengths. The smallest dorsal horn cell RFs are substantially smaller, with contiguous convergence ratios of less than 3 . Therefore the passive convergence model is not adequate to account for the sizes of dorsal horn cell RFs, by a factor of more than 1.5 , and some active process must restrict convergence.

\section{Discussion}

It is clear from Figures 3 and 4 that the terminal fields of axon pairs in the same spinal cord are in consistent somatotopic relation to each other. It is also clear that all the categories of LTCM axons studied obeyed the same ordering rules. At least in a qualitative sense, Figure 6 shows that the somatotopy of primary afferent terminal field projection centers is similar to the somatotopy of dorsal horn cells. Therefore, we suggest that a single map of the skin applies to all LTCMs regardless of receptor type, as well as to all dorsal horn cells regardless of cell type (e.g., local interneuron or cell of origin of an ascending tract). Although there may be subtle quantitative differences in the mapping functions for different afferent or cell types, they cannot be determined with the present sample size.

Shortland et al. (1989) examined the somatotopic organization of hair follicle afferents in the rat dorsal horn, using similar methods to those reported here. They concluded that the projections of these afferents were somatotopically ordered, although they felt that, given the considerable overlap of adjacent terminal fields, the somatotopic organization was not very precise. A pattern of shifting overlap of projections need not imply imprecision, as we have demonstrated in our analysis. However, in the limiting case of very small spinal cords, in small vertebrates, it would not be surprising if the terminal fields of single afferent axons occupy a larger fractional width of the dorsal horn, thus increasing the need for specificity of connections in order to provide tactile acuity.

Our estimated biological error of $\pm 32 \mu \mathrm{m}$ in the topographic ordering of primary afferent projection centers is only a rough approximation based on a single axon pair. It is interesting to note that the widths and lengths of projections of these 2 axons were not in as good agreement as their centers. It will be interesting to see whether analysis of actual bouton densities, in larger numbers of axons, can be used to detect regularities which we miss with this relatively crude type of analysis. This level of precision has important consequences with regard to (a) possible mechanisms of development of the somatotopic map, and (b) the use of assumed bilateral symmetry in plasticity research where 1 side of the dorsal horn is used as a control for the other, experimentally manipulated, side. Specifically, any proposed models of development must account for this level of precision, and attempts to study subtle plastic effects involving changes of less than $10 \%$ of the map width are probably futile with current methods unless averaging across large samples is used.

We do not assume that the precision of projections has important direct implications for the role of dorsal horn in tactile spatial discriminations, because that is presumably determined by the statistics of dorsal horn cell RF geometries and connections to higher centers. Dorsal horn cell RFs are determined by connectivities between afferents and cells and by modulatory influences, and not directly by the locations of cells or afferent terminal fields. However, it is clear that the simplest model of dorsal horn cell RF assembly, passive sampling of presynaptic neuropil by dorsal horn dendrites, must be rejected, at least with regard to the mediolateral axis. The alternative models which must be considered must involve some active process which imparts selectivity to connections. It is already well established that the laminar segregation of terminals of primary afferent submodalities is not adequate to account for submodality specificity of dorsal horn cells. For example, spinocervical tract neurons demonstrate a selectivity in their responsiveness to LTCM afferent types (Brown and Franz, 1969), as do dorsal horn cells in general (Tapper et al., 1973), in spite of the fact that all $A \beta$ LTCMs have terminals that are intermingled in laminae III and IV. Thus, the laminar segregation of afferent terminals and cells in the dorsal horn cannot account for the segregation of LTCM submodalities among the different convergence classes. A similar selectivity must exist with regard to spatial convergence.

Mechanisms underlying such functional specificity can be subdivided into 2 broad classes: anatomical selectivity of actual connections, and "sculpting" of RFs by modulatory influences. Mechanisms in the dorsal horn underlying selective connections, either to mediate specificity of submodality convergence or to control RF size, are not understood. However, a number of mechanisms have been proposed for modulation of RF size.

One factor that could contribute to the determination of RF size would be the presence of mechanisms that render specific synapses ineffective (e.g., Wall et al., 1967; Merrill and Wall, 1972; Basbaum and Wall, 1976; Wall and Werman, 1976; Wall, 1977; Devor and Wall, 1981; Koerber and Brown, 1982). These may be held ineffective by presynaptic inhibition or neuromodulatory influences, or by other factors that prevent invasion of the terminals by action potentials or prevent the release of transmitter. Alternatively, postsynaptic elements may be unresponsive to part of their inputs as a result of, e.g., postsynaptic membrane properties at selected synaptic sites or in selected dendritic branches. Finally, it is conceivable that postsynaptic inhibition may play a role in localized modulation of postsynaptic responsiveness. Whatever the mechanism, a spectrum of possibilities exists between 2 extremes: (a) Some ineffective synapses are held ineffective at all times, except during plastic responses to injury, or (b) all synapses are effective at one time or another, depending on the physiological state of the animal, and some are ineffective some of the time.

The somatotopic organization of primary afferent projections to cat dorsal horn is precise in terms of the locations of projection centers. Although projection areas may be small enough to mediate the largest RFs seen in dorsal horn cells by passive assembly, they are too large for passive assembly of average or smaller than average dorsal horn cell RFs. If further studies of axon pairs reveal that the precision of projection centers is of the order of $32 \mu \mathrm{m}$, it is reasonable to ask why the projection centers are so precise, but the projection areas are relatively large. The answer must lie in the developmental processes that led to the adult organization. One of many possibilities is that axons initially project to even larger areas of the dorsal horn, and dorsal horn cell dendrites initially extend over larger areas as well. If a competitive mechanism is used to form the somatotopic map (c.g., Hcbb, 1949; Willshaw and von der Malsburg, 1976), then axons and dendrites may retract as RFs shrink during the selforganization process. In that case, a relatively diffuse morphology (relatively large axonal arbors and dendritic trees) could still provide the anatomical substrate for a relative precise physiology (relatively restricted divergence and convergence, and relatively small dorsal horn cell RFs). In fact, a diffuse morphology is to be expected, because occasional contacts between 
distal axonal branches and distal dendrites will be retained because, by chance, they will contribute to the final dorsal horn cell RFs.

If axons do terminate outside the zone of responsive cells, yet still form effective connections, then the presynaptic somatotopy hypotheses of Brown and Fuchs (1975) should be modified to predict that axons project not just to the zone of responding cells, but to the zone defined by the combined dendritic span of such cells. This zone could extend a few hundred micrometers mediolaterally and a few millimeters rostrocaudally, judging from the reported anatomy of dorsal horn cell dendrites (e.g., A. G. Brown et al., 1977b; Proshansky and Egger, 1977; Brown and Fyffe, 1981).

Although some investigators have concluded that individual axons project outside the zone of responding dorsal horn cells (somatotopically inappropriate connections: e.g., Meyers and Snow, 1984), and therefore that some of the afferent connections must be normally ineffective, their studies have not excluded 2 important alternative possibilities: (a) The zone of responding cells may be underestimated because of the presence of undetected responding cells, such as the small neurons known to exist in all laminae, cells in deeper laminae that receive LTCM input via their dorsal dendrites, cells that respond under physiological conditions other than those explored, or scattered cells with unusually large RFs. (b) All of an axon's connections can be effective, if they are restricted to dendrites of responding cells, and the dendrites extend sufficiently far from their cell bodies. Until these possibilities have been excluded, the conclusion that some projections are somatotopically inappropriate is premature.

\section{References}

Adams JC (1977) Technical considerations on the use of horseradish peroxidase as a neuronal marker. Neuroscience 2:141-145.

Basbaum AI, Wall PD (1976) Chronic changes in the response of cells in adult cat dorsal horn following a partial deafferentation. The appearance of responding cells in a previously non-responsive region. Brain Res 116:181-204.

Brown AG, Franz DN (1969) Responses of spinocervical tract neurones to natural stimulation of identified cutaneous receptors. Exp Brain Res 7:231-249.

Brown AG, Fyffe REW (1981) Form and function of spinal neurones with axons ascending the dorsal columns in the cat. J Physiol (Lond) 321:31-47.

Brown AG, Rose PK, Snow PJ (1977a) The morphology of hair follicle afferent collaterals in the spinal cord of the cat. J Physiol (Lond) 272: 779-797.

Brown AG, Rose PK, Snow PJ (1977b) The morphology of spinocervical tract neurones revealed by intracellular injection of horseradish peroxidase. J Physiol (Lond) 270:747-764.

Brown AG, Rose PK, Snow PJ (1978) Morphology and organization of axon collaterals from afferent fibres of slowly adapting type I units in cat spinal cord. J Physiol (Lond) 277:15-27.

Brown AG, Fyffe REW, Noble R (1980a) Projections from Pacinian corpuscles and rapidly adapting mechanoreceptors of glabrous skin to the cat's spinal cord. J Physiol (Lond) 307:385-400.

Brown AG, Fyffe REW, Noble R, Rose PK, Snow PJ (1980b) The density, distribution, and topographical organization of spinocervical tract neurones in the cat. J Physiol (Lond) 300:409-429.

Brown AG, Fyffe REW, Rose PK, Snow PJ (1981) Spinal cord collaterals from axons of Type II slowly adapting units in the cat. J Physiol (Lond) 316:469-480.

Brown PB (1986) DORSALVIEw: a program which displays horizontal views of histological material in transverse spinal cord sections. J Electrophysiol Techs 13:27-42.

Brown PB, Culberson JL (1981) Somatotopic organization of hindlimb cutaneous dorsal root projections to cat dorsal horn. J Neurophysiol 45:137-143.
Brown PB, Fuchs JL (1975) Somatotopic representation of hindlimb skin in cat dorsal horn. J Neurophysiol 38:1-9.

Brown PB, Koerber HR (1978) Cat hindlimb tactile dermatomes determined with single-unit recordings. J Neurophysiol 41:260-267.

Brown PB, Ritz LA (1986) Dorsal horn potentials and current source densities evoked by single action potentials in single slowly adapting Type I axons. J Neurophysiol 55:1104-1 113

Brown PB, Millecchia RJ (1987) A sophisticated microcomputer-based laboratory controller: II. Host software. J Electrophysiol Techs 14: $13-41$.

Brown PB, Fuchs JL, Tapper DN (1975) Parametric studies of dorsal horn neurons responding to tactile stimulation. J Neurophysiol 38: 19-25.

Brown P, Sonty R, Millecchia R, Gladfelter W, Culberson J (1988) Somatotopy of cutaneous axons in cat dorsal horn laminae III-IV: qualitative. Soc Neurosci Abstr 14:693.

Brown PB, Koerber HR, Hobbs GR (1990) Precision of cat dorsal horn somatotopic map. Soc Neurosci Abstr 16:560.

Bryan RN, Trevino DL, Coulter JD, Willis WD (1973) Location and somatotopic organization of the cells of origin of the spinocervical tract. Exptl Brain Res 17:177-189.

Devor M, Wall PD (1981) Effect of peripheral nerve injury on receptive fields of cells in the cat spinal cord. J Comp Neurol 199:277-291.

Hebb DO (1949) Organization of behavior. New York: Wiley.

Horch KW, Tuckett RP, Burgess PR (1977) A key to the classification of cutaneous mechanoreceptors. J Invest Dermatol 69:75-82.

Koerber HR, Brown PB (1980) Projections of two hindlimb nerves to cat dorsal horn. J Neurophysiol 44:259-269.

Koerber HR, Brown PB (1982) Somatotopic organization of hindlimb cutaneous nerve projections to cat dorsal horn. J Neurophysiol 48: $481-489$.

Koerber HR, Brown PB, Mendell LM (1990) Correlation of rostrocaudal distributions of field potential evoked by single action potentials in identified cutaneous afferents and the rostrocaudal distributions of their terminals. J Comp Neurol 294:133-144.

Light AR, Durkovic RG (1984) Features of laminar and somatotopic organization of lumbar spinal cord units receiving cutaneous inputs from hindlimb receptive fields. J Neurophysiol 52:449-458.

Maxwell DJ, Bannatyne BA, Fyffe REW, Brown AG (1982) Fine structure of hair follicle afferent fibre terminations in the spinal cord of the cat. J Neurocytol 11:571-582.

Merrill EG, Wall PD (1972) Factors forming the edge of a receptive field: the presence of relatively ineffective afferents. J Physiol (Lond) 226:825-846.

Meyers DER, Snow PJ (1984) Somatotopically inappropriate projections of single hair follicle afferent fibres to the cat spinal cord. $J$ Physiol (Lond) 347:59-73.

Millecchia RJ, Brown PB (1986) A sophisticated microcomputer-based laboratory controller: I. Hardware and firmware. J Electrophysiol Techs 13:161-187.

Proshansky E, Egger MD (1977) Dendritic spread of dorsal horn neurons in cats. Exp Brain Res 28:153-166.

Pubols BH, Hirata H, West-Johnsrud L (1989) Somatotopic organization of forelimb representation in cervical enlargement of raccoon dorsal horn. J Neurophysiol 61:126-137.

Ritz LA, Culberson JL, Brown PB (1985) Somatotopic organization in spinal cord segments with fused dorsal horns: caudal and thoracic levels. J Neurophysiol 54:1167-1177.

Ritz LA, Brown PB, Bailey SM (1989) Crossed and uncrossed projections to cat sacrocaudal spinal cord: I. Axons from cutaneous receptors. J Comp Neurol 289:284-293.

Shortland P, Woolf CJ, Fitzgerald M (1989) Morphology and somatotopic organization of the central terminals of hindlimb hair follicle afferents in the rat lumbar spinal cord. J Comp Neurol 289:416433

Sonty R, Brown P, Millecchia R, Gladfelter W, Culberson J (1988) Somatotopy of cutaneous axons in cat dorsal horn laminae III-IV: quantitative. Soc Neurosci Abstr 14:693.

Tapper DN, Brown PB, Moraff H (1973) Functional organization of the cat's dorsal hom: connectivity of myelinated fiber systems of hairy skin. J Neurophysiol 36:817-826.

Wall PD (1960) Cord cells responding to touch, damage, and temperature of skin. J Neurophysiol 23:197-210.

Wall PD (1977) The presence of ineffective synapses and the circumstances which unmask them. Phil Trans Roy Soc Lond [Biol] 278: $361-372$. 
Wall PD, Werman R (1976) The physiology and anatomy of long ranging afferents fibres within the spinal cord. J Physiol (Lond) 255: 321-334.

Wall PD, Freeman J, Major D (1967) Dorsal horn cells in spinal and freely moving rats. Exp Neurol 19:519-529.

Willshaw DJ, von der Malsburg C (1976) How patterned neural con- nections can be set up by self-organization. Proc Roy Soc Lond [Biol] 194:431-445.

Wilson P, Meyers DER, Snow PJ (1986) The detailed somatotopic organization of the dorsal horn in the lumbosacral enlargement of the cat spinal cord. J Neurophysiol 55:604-617. 